\title{
Octopus-Inspired Innovative Suction Cups
}

\author{
Maurizio Follador ${ }^{1,2}$, Francesca Tramacere ${ }^{1,2}$, Lucie Viry $^{1}$, Matteo Cianchetti ${ }^{2}$, \\ Lucia Beccai $^{1}$, Cecila Laschi ${ }^{2}$, and Barbara Mazzolai ${ }^{1}$ \\ 1 Center for Micro-BioRobotics@SSSA, Istituto Italiano di Tecnologia, \\ Pontedera, Italy \\ 2 The BioRobotics Institute, Scuola Superiore Sant'Anna, Pisa, Italy \\ Maurizio.Follador@iit.it
}

\begin{abstract}
Octopus show great adhesion capabilities thanks to their suckers covering their ventral side of their arms. Starting from biological investigation, we identified preliminary specifications for the design of innovative artificial suction cups, which could be used in the field of soft robotics. The main features of the biological sucker are maintained as leading criteria for the choice of the actuation technology and mechanism. In this preliminary work, we focused on the imitation of the functionality of the specific muscle bundles which generate suction to obtain adhesion. Dielectric Elastomers Actuators (DEA) were identified as a suitable solution. A study on materials and manufacturing techniques was made. Different possible solutions in the use of DEA are also described.
\end{abstract}

Keywords: bioinspired wet adhesion, dielectric elastomers, soft robotics.

\section{Functional Features of Octopus Suckers}

Octopus sucker is a muscular hydrostat which consists of two main portions, an upper hollow cup, the acetabulum, and a lower disk-like portion, the infundibulum. The two portions are connected by means of an orifice. The musculature arrangement of octopus sucker consists of three kinds of fibers (Fig. 1(a)): radial fibers ( $\mathrm{r}$ ) which extend throughout the thickness of the sucker; circular fibers (ci) which are oriented parallel to the opening surface of the infundibulum; and meridional fibers $(\mathrm{m})$ which extend from the apex to the bottom of the whole sucker. In order to obtain adhesion, a seal is formed thanks to the rim which encircles the infundibulum, and then a the pressure differential is created in between the internal cavity of the cup and the outside, by the contraction of the radial muscles. Circular and meridional muscles are instead involved in the detachment mechanism 1 .

\section{Specifications for the Artificial Sucker}

The study of the biological sucker gives inputs for the definition of the design specifications for the performances expected in the suction cup inspired by the 


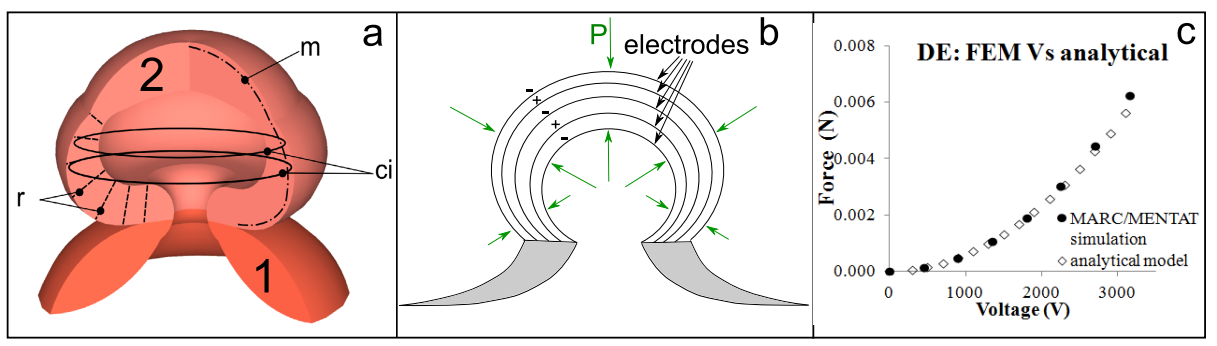

Fig. 1. (a) 3D CAD model of the octopus sucker 1- infundibulum 2-acetabulum r- radial muscles ci- circular mmuscles m- meridional muscles. (b) concept for the use of DEA, longitudinal section P- radial pressure generated by DEA. (c) Force-Voltage graph of actuator in planar stretching, analytical and FEM model comparison, ref. Sect. 3.1

natural counterpart. Octopus suckers are able to generate a differential pressure up to $0.27 \mathrm{MPa}$, in the time scale of milliseconds. This specifications was used to identify the most suitable technology for the realization of the bioinspired artefact. At this preliminary stage, we focused on the role of the radial muscles, which produce the differential pressure for the adhesion. The characteristics needed for this particular application are: good compliance, for the integration in a soft structure, capability to work in wet conditions and possibility of miniaturization. We identified dielectric elastomers as actuators able to mimic the contraction effect performed by radial muscles. The actuators developed, described in Sect. 3.1, can generate an internal stress up to 0.4 MPa with a speed of hundreds of milliseconds.

\section{Design of the Artificial Sucker}

Dielectric elastomers represent a good candidate for the characteristics mentioned in Sect. 2, Moreover the fabrication procedure makes them suitable for the design of actuators with different shapes and geometries. In presence of an electric field, the dielectric membrane is subjected to a stress field described by the Maxwell stress tensor:

$$
\sigma_{M}=\varepsilon E \otimes E-\frac{\varepsilon(E \cdot E) I}{2} .
$$

where $E$ is the electric field, $I$ is the identity tensor and $\varepsilon$ is the dielectric constant of the material. In the proposed solution, the actuator is composed by many dielectric elastomer layers, as represented in Fig. 1(b), which compose the acetabular portion of the suction cup. When the voltage is applied, the electrodes are attracted to each other, inducing a reduction of the thickness of the acetabular wall and an increase of the volume enclosed. If the rim of the infundibulum is sealed on the substrate, the volume of water inside the sucker remains constant. In this configuration, the isometric contraction of the actuator reduces the pressure of the internal fluid and adhesion force is generated. 


\subsection{Experimental Results}

A commercial Fluorosilicone (Dow Corning 730) was selected for its dielectric properties [2. This dielectric material is spin-coated to obtain a layer of $70-$ $100 \mu \mathrm{m}$. The electrode, made of carbon black, is sprayed on the silicone surface after suspension in a solvent [3]. A procedure for the fabrication of multilayer actuators, in a fast and repeatable way, was defined. It consists of the following main steps: (i) the dielectric layer is spin-coated; (ii) the electrode is sprayed on it just before complete curing of the silicone; (iii) a second layer is spin-coated on the previous one. This steps are repeated to obtain the desired number of layers. The design of the actuator, as shown in Fig. 1(b), could present some issues in sustaining the pressure difference, due to the compliance of the actuator. In order to overcome this limit, we consider to embed an elastic element, with the aim to support the silicone active membrane and accumulate elastic energy. This passive elements work as antagonist respect to the membrane. When the actuator is in rest position it constrains the elastic element, which stores energy, that is released when the actuator is activated. A FEM model was developed in Marc/Mentat (Msc Software, CA, USA) to evaluate the performances of the actuators, the different geometries and the interaction with the rest of the soft structure. A good correspondence between the analytical model (1) and the simulation was found and shown in Fig. 1(c). The force produced by a rectangular actuator undergoing planar stretching in an isometric actuation, as illustrated in 4, was calculated with both methods and compared.

\section{Discussion and Future Work}

This work is the initial step through the design of an active suction cup bioinspired by the octopus sucker. This preliminary study on the capabilities of dielectric elastomers, demonstrates the possibility to fabricate an actuator that mimics the functionality of octopus sucker radial muscles. The use of FEM models will be used in the design process of the actuators in relation with the other part of the structure, which, for design specifications, are totally soft. The artificial sucker prototypes will be designed starting from the reconstruction of the biological sucker (Fig. 1(a)), embedding the actuated parts in the acetabulum.

\section{References}

1. Kier, W.M., Smith, A.M.: The Structure and Adhesive Mechanism of Octopus Suckers, 1153, 1146-1153 (2002)

2. Pelrine, R., Kornbluh, R., Joseph, J., Heydt, R., Pei, Q., Chiba, S.: High-field deformation of elastomeric dielectrics for actuators. Materials Science and Engineering: C 11(2), 89-100 (2000)

3. Carpi, F., Migliore, A., Serra, G., De Rossi, D.: Helical dielectric elastomer actuators. Smart Materials and Structures 14(6), 1210 (2005)

4. Kofod, G., Sommer-Larsen, P.: Silicone dielectric elastomer actuators: Finiteelasticity model of actuation. Sensors and Actuators A: Physical 122(2), 273-283 (2005) 